\title{
Pyoderma gangrenosum associated with acute myeloproliferative disease (? erythroleukaemia)
}

\author{
Francis ToOLIS \\ M.R.C.P. (U.K.)
}

\author{
Alistair C. Parker \\ Ph.D., M.R.C.Path.
}

\begin{abstract}
Summary
Pyoderma gangrenosum at the site of sternal marrow aspirate was observed in a patient with acute myeloproliferative disease characterized by a high peripheral blood basophilia. Lesions with a similar appearance were also seen on the palate. No other disease known to be associated with pyoderma gangrenosum could be demonstrated. A review of the literature suggests that myeloproliferative disease should now be recognized as occurring in association with pyoderma gangrenosum.
\end{abstract}

\section{Introduction}

Pyoderma gangrenosum is a rare skin disorder which typically takes the form of one or more plaques, each with an area of central necrosis delineated by a characteristic bluish-red border. Although most commonly reported (Domonkos, 1971) in association with ulcerative colitis and rheumatoid arthritis, pyoderma gangrenosum has also been noted in other clinical syndromes including acute and chronic myeloproliferative disease (Perry and Winkelmann, 1972; Shore, 1976; Cramers, 1976). The authors now describe a patient with an acute myeloproliferative disorder characterized by a pronounced and persistent peripheral blood basophilia and the occurrence of pyoderma gangrenosum initiated by sternal marrow puncture.

\section{Case report}

A previously healthy 53 -year-old salesman gave a 12-month history of malaise, weight-loss, night sweats and recurrent respiratory tract infections. There was a family history of diabetes mellitus, his mother being a late-onset, insulin-dependent diabetic. Initial clinical examination revealed generalized pallor, hepatomegaly of $5 \mathrm{~cm}$ and a spleen just palpable at the costal margin.

The initial haemoglobin concentration was $10 \cdot 1$ $\mathrm{g} / \mathrm{dl}$, with a platelet count of $64 \times 10^{9} / 1$, a white cell count of $12.4 \times 10^{9} / 1$ (differential: $19 \%$ neutrophils, $20 \%$ lymphocytes, $10 \%$ monocytes, $19 \%$ eosinophils, $22 \%$ basophils, $4 \%$ myelocytes, $4 \%$ promyelocytes and $2 \%$ blast cells), a reticulocyte count of
$6.5 \%$, erythrocyte sedimentation rate of $15 \mathrm{~mm} /$ hour and additionally two nucleated red cells per $100 \mathrm{G}$. white cells were noted. The blood film showed baso- $+\uparrow$ philic stippling, neutrophil toxic granulation and $\mathcal{E}_{\mathcal{O}}$ pseudo-Pelgerization. The leucocyte alkaline phos- $-\infty$ phatase score was 19 (normal range 20-100). ట్ర Sternal marrow aspirate, obtained with difficulty, $\mathrm{O}$ showed megaloblastic erythropoiesis, an increase in promyelocytes and blast cells, numerous bizarre $\vec{\square}$

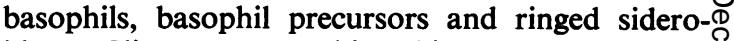
blasts. Iliac crest trephine biopsy demonstrated $\stackrel{\Phi}{\mathcal{S}}$ hypercellularity with a prominence of blast ceㅍㅎㅇ and excess reticulin. The serum $B_{12}$ was greater than $1000 \mathrm{ng} / \mathrm{l}$ (normal range 170-7000 ng/l) and the $\overrightarrow{0}$ serum folate and Schilling test were normal.

Stools were repeatedly strongly positive to testipg for faecal occult blood, but barium studies of the gastrointestinal tract were normal and no abnor- $-\frac{5}{0}$ mality of haemostasis other than thrombocytopenia@ could be demonstrated. There was no evidence of

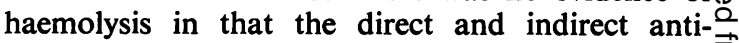
globulin tests were negative, there was no urinary을 haemosiderin, and the plasma haemoglobin, serum methaemalbumin and plasma haptoglobin were within normal limits.

Initial treatment was blood transfusion. In addition, in view of his symptoms and the presence of a strongly positive Mantoux test, a diagnosis of 3 . occult tuberculosis was considered and a trial of ethambutol and isoniazid begun. Although night sweats and malaise diminished, bacteriological ${ }_{\circ}$ confirmation of tuberculosis was never obtained despite culture of urine, sputum and marrow.

On follow-up, utilizing standard surgical techniques and thiomersal as a topical antiseptic, a $N$ repeat sternal marrow aspiration was attempted but $N$ was a dry tap. One week later a large, painful, red N swelling developed in the skin at the site of ${ }_{\sigma}^{\omega}$ aspiration. This lesion developed central necrosis and when the necrotic area was removed the ulcer $O$ had a cribriform base with a ragged bluish-red $\stackrel{\infty}{\oplus}$ overhanging edge characteristic of pyoderma ${ }_{+}^{+}$ gangrenosum. This lesion slowly extended in size to 


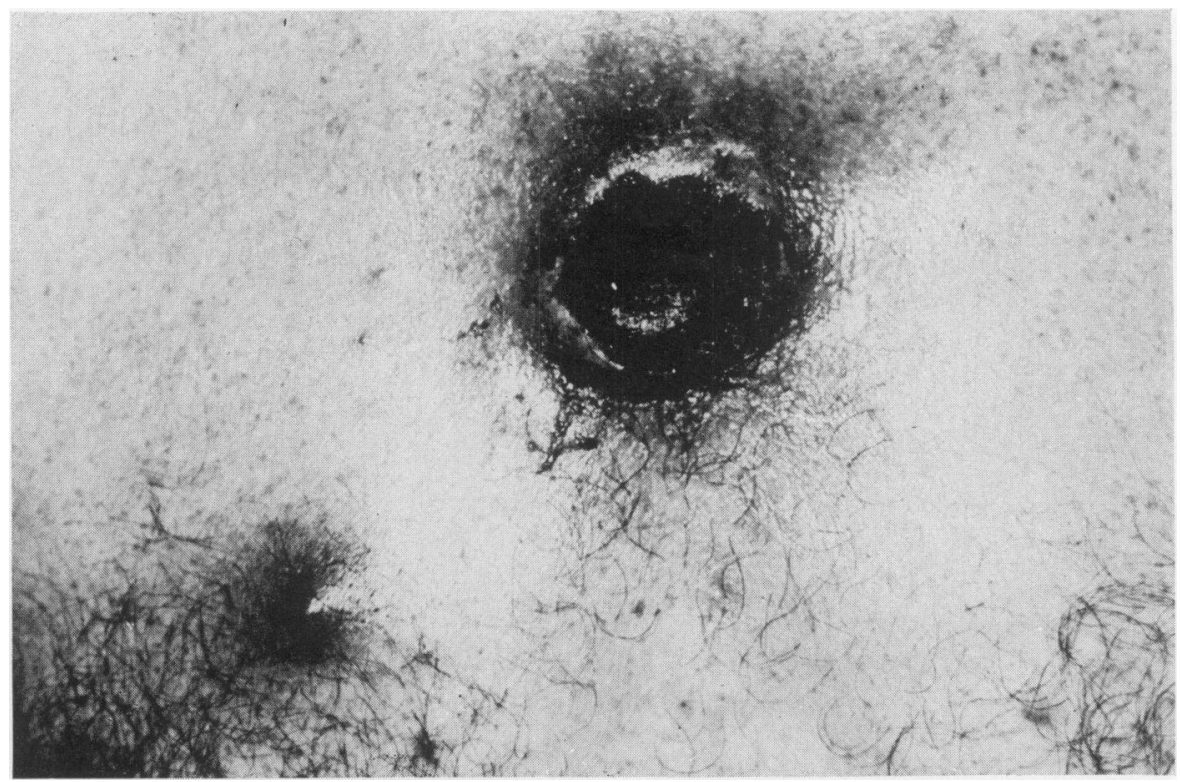

Fig. 1. This illustrates the initial lesion of pyoderma gangrenosum over the sternum, and lower left is the second lesion at early stage of development.

reach a diameter of $5 \mathrm{~cm}$. Later a second painful swelling, identical in appearance to the first, developed near the first lesion (Fig. 1). Biopsy of this second area showed an infiltrate of lymphocytic cells in the epidermis and around the dermal blood vessels, while bacteriological studies revealed Staphylococcus aureus but no anaerobic organisms. The pyoderma gangrenosum responded rapidly to $60 \mathrm{mg}$ prednisolone/day, but mild diabetes mellitus, controlled well by diet and chlorpropamide, developed.

On further investigation at this time the following results were negative or within the normal range: urea and electrolytes, liver function tests, calcium, phosphate, uric acid, serum proteins, protein electrophoresis, immunoglobulins, cryoglobulins, serum viscosity, anti-nuclear factor, LE cell preparation, rheumatoid arthritis latex test, ASO titre, VDRL flocculation test, TPHA test, prothrombin time ratio, PTTK, fibrinogen, fibrin degradation products ethanol gelation test, prothrombin consumption index, serum lysozyme, neutrophil function tests, skin antibody immunofluorescence tests and $\mathrm{CH}_{50}$ and $\mathrm{C}_{4}$ levels, while the $\mathrm{C}_{3}$ level was slightly elevated at $2.74 \mathrm{~g} / 1$ (normal range $0.85-2.54 \mathrm{~g} / \mathrm{l}$ ).

The patient appeared to improve subjectively on the treatment previously outlined with an increase in his sense of well-being and reduction in night sweats. His steroid dosage was gradually reduced to $10 \mathrm{mg} /$ day, but he then developed two necrotic areas in the palate closely resembling the pyoderma gangrenosum present on his chest (Fig. 2). No significant bacterial infection could be demonstrated in these lesions.

He became more anaemic, requiring further blood transfusions which eventually totalled 34 units in the 4 months after presentation, and severely thrombocytopenic (platelet count $12 \times 10^{9} / 1$ ), resulting in purpura and recurrent epistaxes. His total white blood count remained within normal limits, but the basophil count fluctuated markedly, reaching more than $40 \%$ of the total white cell count on several occasions. Myeloid precursors were invariably present in the peripheral blood count, but the blast cell count never exceeded $5 \%$.

A trial of oxymetholone produced no clinical improvement or reduction in transfusion requirement, and eventually the patient died 4 months following his initial presentation. A post-mortem failed to reveal any known association of pyoderma gangrenosum. The marrow showed intense erythroid hyperplasia, normoblastic in type and negative to periodic acid-Schiff staining, with reduction in all other cell lines. Whilst no firm diagnosis could be reached the overall appearances were considered to be consistent with erythroleukaemia. 


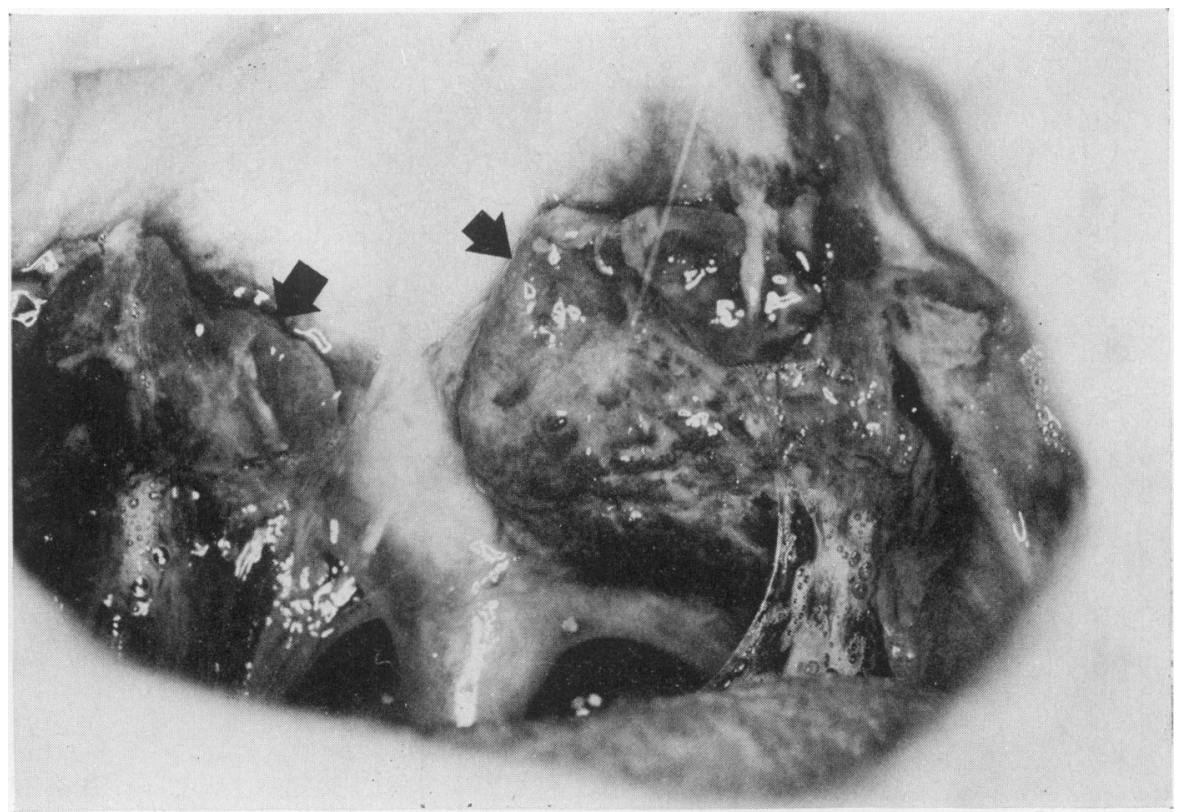

Fig. 2. This illustrates two large ulcerated areas on palate resembling pyoderma gangrenosum.

\section{Discussion}

Pyoderma gangrenosum has been reported in association with ulcerative colitis, rheumatoid arthritis, regional ileitis, dermatitis herpetiformis, hypogammaglobulinaemia, paraproteinaemia and myeloma (Domonkos, 1971). Other associations include active chronic hepatitis (Byrne, Hewitt and Summerly, 1976), immunosuppression (Haim et al., 1973) and disseminated intravascular coagulation (Staughton and Copeman, 1976). Alterations of cellular immunity have also been noted (Shore, 1976). Pyoderma gangrenosum has occurred in a case of acute lymphoblastic leukaemia maintained in remission by prednisolone, methotrexate and 6mercaptopurine, whilst non-specific leukaemia has occurred following 6-mercaptopurine, for preexisting pyoderma gangrenosum (Shore, 1976).

There have been a number of reports in the literature of this rare skin condition occurring in association with myeloproliferative diseases, including acute myeloblastic leukaemia, myelofibrosis, chronic myeloid leukaemia, polycythaemia rubra vera and acute myelomonocytic leukaemia (Perry and Winkelmann, 1972; Shore, 1976; Cramers, 1976). Few of these reports, however, were fully documented and the authors were fortunate in being able to investigate their patient extensively. Nevertheless, no definitive haematological diagnosis was possible but the initial marrow findings together with the abnormal peripheral blood, the rapid progression of the disease and the post-mortem findings suggest that the disorder could be classified within tha myeloproliferative spectrum as defined by Gunz are $\overrightarrow{0}$ Baikie (1974), and was acute in type. A peculiar $\infty$ feature was the persistent peripheral blood bas $\hat{\sigma}^{-}$ philia. Whether the pyoderma gangrenosum and the basophilia were causally related is open to speculation.

Finally, accepting the fact that there is no satis- $\frac{\mathscr{Q}}{\varnothing}$ factory means of causally linking the skin lesions and the underlying haematological disorder, the nature $\overrightarrow{\overrightarrow{0}}$ of the pyoderma gangrenosum in this case was unusual in two respects. The primary lesion developed on the site of a recent sternal marrow puncture suggesting that direct trauma by some unknown means precipitated the epidermal necrosis. Secondly, the patient had lesions resembling pyoderma gangrenosum on the palate, a site of involvement which has rarely, if ever, been implicated.

\section{Acknowledgments}

We wish to thank Dr S. H. Davies for permission to report his patient, Dr S. Urbaniak of the Blood Transfusion Service for undertaking the neutrophil function studies and complement levels, Dr H. White and Dr J. D. McGregor of N the Pathology Department of the University of Edinburgh, and Miss J. Donnelly for typing the manuscript.

\section{References}

Byrne, J.P.H., Hewitt, M. \& Summerly, R. (1976) Pyoderma gangrenosum associated with active chronic hepatitis. Archives of Dermatology, 112, 1297.

Cramers, M. (1976) Bullous pyoderma gangrenosum in association with myeloid leukaemia. Acta dermatovenereologica, 56, 311 . 
Domonkos, A.N. (1971) Andrews' Diseases of the Skin, p. 926. W. B. Saunders, Philadelphia.

Gunz, F. \& BAIKIE, A. (1974) Leukemia, 3rd edn. Grune \& Stratton, New York.

Haim, S., Friedman-Birbaum, R., Better, O.S. \& Tuma, S. (1973) Skin complications in immunosuppressed patients; follow-up of kidney recipients. British Journal of Dermatology, 89, 169.
Perry, H.O. \& WinkelmanN, R.K. (1972) Bullous pyoderma gangrenosum and leukemia. Archives of Dermatology, 106, 901.

ShORE, R.N., (1976) Pyoderma gangrenosum, defective neutrophil chemotaxis, and leukemia. Archives of Dermatology, 112, 1792.

Staughton, R.C.D. \& Copeman, P.W.M. (1976) Chronic intravascular coagulopathy with? pyoderma gangrenosum. British Journal of Dermatology, 95 (Suppl. 14), 70. 